\title{
Chitosan-Tripoly Phosphate (CS-TPP) Synthesis Through Cross-linking Process: The Effect of Concentration Towards Membrane Mechanical Characteristic and Urea Permeation
}

\author{
RETNO ARIADI LUSIANA ${ }^{1 *}$, WAHYU PUTRI PROTONINGTYAS ${ }^{1}$, ANUGRAH RICKY \\ WIJAYA $^{2}$, DWI SISWANTA ${ }^{3}$, MUDASIR $^{3 * \star}$ and SRI JUARI SANTOSA ${ }^{3}$ \\ ${ }^{1}$ Chemistry Department, Faculty of Sciences and Mathematics, Diponegoro University, \\ Semarang, Indonesia. \\ ${ }^{2}$ Chemistry Department, Faculty Mathematics and Natural Sciences, Universitas Negeri Malang \\ (UM) or State University of Malang, Indonesia. \\ ${ }^{3}$ Chemistry Department, Faculty of Mathematics and Natural Sciences, Gadjah Mada University, \\ Yogyakarta, Indonesia. \\ *Corresponding author E-mail: retno.lusiana@live.undip.ac.id, mudasir@ugm.ac.id
}

http://dx.doi.org/10.13005/ojc/330626

(Received: August 12, 2017; Accepted: September 10, 2017)

\begin{abstract}
Crosslinking reactions are required to modify the functional groups on chitosan and to increase the number of active groups of chitosan. In this study crosslinking reaction of tripolyphospate (TPP) groups on chitosan was conducted in order to produce membrane which functions as candidate of hemodialysis membrane. The mole ratio between chitosan and NaTPP was varied to obtain the best composition of modified materials. Study of TPP concentration effect towards membrane characteristics was conducte durea permeation ability. The resulting membrane was then characterized including tests of thickness, water uptake, tensile strength and membrane hydrophilicity. The results showed that the best composition of membrane occurred in the addition of $0.691 \mathrm{~g}$ of NaTPP proved byin creasing water uptake $(150 \%)$, its highest mechanical strength and releasing the percentage of urea permeation by $44 \%$.
\end{abstract}

Keywords: Crosslinking, Membrane mechanical, Urea permeated.

\section{INTRODUCTION}

Membrane technology is developing rapidly and refining its method continuosly. Membrane is a thin semi permeable and selective layer that can restrain specific components and pass other components based on diffusion coefficient, electrical charge, and solubility differences'. Membrane composition and material structure are the most important components in membrane based technology $y^{2-3}$. The increasing use of membrane in various industries is not separated from the membrane's advantages, such as membrane works with sieving mechanism principal, solution diffusion, and no need additive chemical compounds. It can isothermally operate in in room temperature and consumes low energy ${ }^{4}$. 
One of the basic components of membrane forming process is chitosan. Chitosan membranes are becoming widely used in medical field such as hemodialysis. In hemodialysis process, membrane replaces kidney's function to remove the remaining metabolism compounds such as urea, creatinine, and glucose outside the body through urine ${ }^{5}$.

In its use as hemodialysis membrane, there are some characteristics that must be possessed by a membrane, for instance, evenly small pores, no blood rejection, mechanically strong, has active groups which function aspermeate catcher through hydrogen bond formation and reactive ${ }^{6}$.

Chitosan is a chitin derivative which isopolyatomic, nontoxic, low immunogenic, biocompatible, biodegradable, and in weak acid can form thin layer of membrane. Chitosan's biodegradable characteristic makes this compound as a recommendation for application use in environmentally friendly industry ${ }^{7}$. From the process, the forming of chitosan membrane is relatively simple and needs less time than the forming of synthetic membrane. In the other side, chitosan based on membrane alone has some disadvantages, which are: less porous membrane, deficient mechanical characteristic (fragile), weak resistance while pulled and strained, and lack of active sides that function as binder agent of the target compound ${ }^{8}$.

In addition, positive charge of chitosan amine groups can interact with protein which causes protein adsorption on the membrane surface ${ }^{7}$. If this process was left alone, there would be fouling membrane that caused the membrane became no longer be able to function in permeation process ${ }^{9}$.

Modification towards material or chitosan membrane surface through chemical process and the blending with other synthetic polymer nonchitosan is expected to produce membrane with better characteristics. On the other hand, modification is also aimed to enhance the reactivity and mechanical strength of chitosan membrane ${ }^{10}$. A portion of chitosan undergoes extensive crosslinking to give modifies chitosan, whose properties are much different from those of pure chitosan. Nevertheless, polymer crosslinking leads to the formation of permanent covalent network, which may allow the free diffusion of water/bioactive materials and also enhances the mechanical properties of the polymer ${ }^{11}$.

Sodium tripoly-phosphate, an ionic cross linker, having three phosphate groups are can facilitate kinetically driven cross-linking of chitosan in aqueous medium under physiological condition ${ }^{12}$. However, the effects of cross-linked TPP reactions on the physical, chemical and transporting properties of chitosan membranes have not been studied more broadly.

Urea is an important metabolic by-product in living system and the high level of urea on the blood indicates the decreased of the kidney function. Therefore, the uptake of urea in blood is an important process when hemodialysis is required to decrease the urea levels in blood plasma patient with chronic kidney disease ${ }^{12}$.

Considering the background above, crosslinking reactions of tripolyphospate towards chitosan on several concentrations were conducted at a controlled temperature through (-NH-O- $\left.-\mathrm{PO}_{2}-\right)$ bond. The success of cross-linking reaction would be analyzed using FTIR, XPS. Membrane's mechanical strength was enhanced through blending by mixing PVA-PEG mix. This blend was aimed to obtain the proper balance of hydrophilic groups and hydrophobic groups.

In a proper balance, it resulted to a membrane with optimal mechanical strength. This study reports the effect of tripolyphosphate concentration towards membrane characteristics such as water uptake, tensile strength, hydrophilicity, and membrane application in urea transport.

\section{EXPERIMENTAL}

\section{Preparation of chitosan and NaTPP cross-linking reaction}

A few grams of chitosan was weight and diluted into $90 \mathrm{~mL}$ of $1 \%$ acetate acid, added with $1 \mathrm{~mL}$ of concentrated acetate acid and stirred for 4 hours. After that, the solution was added with a few NaTPP dissolved in $9 \mathrm{~mL}$ of $1 \%$ acetate acid by dropping it while stirring the solution (Table 1). 
Reaction temperature was set into range $60-80^{\circ} \mathrm{C}$ for approximately 4 hours. Then the resulted solution was cooled at room temperature while being stirred to refine the reaction and kept as membrane solution (CS-TPP).

Table.1: Chitosan and NaTPP ratio comparison

\begin{tabular}{lcc}
\hline Materials type & Chitosan $(\mathrm{g})$ & NaTPP $(\mathrm{g})$ \\
\hline CS & 1.5 & 0.0000 \\
CS-TPP1 & 1.5 & 0.6901 \\
CS-TPP2 & 1.5 & 0.2381 \\
CS-TPP3 & 1.5 & 0.1735 \\
CS-TPP4 & 1.5 & 0.0863 \\
\hline
\end{tabular}

Preparation CS-TPP and CS-TPP/PVA-PEG blend membranes

$5 \mathrm{~mL}$ of CS-TPP solution was poured into petri dish, and dried in an oven for $24 \mathrm{~h}$ at $50-70^{\circ} \mathrm{C}$. After the membrane dried, $2 \mathrm{M} \mathrm{NaOH}$ was poured into petri dish and washed intensively using aquades to be neutral.

\section{Membrane characterization}

We used FTIR in 400-4000 $\mathrm{cm}^{-1}$ wavelength Schimadzu FTIR spectroscope to identify the chemical compounds of the membrane before and after cross-linking reaction. Thermal degradation behavior of membranes was evaluated in temperature range $30-900^{\circ} \mathrm{C}$ with Linseiss TGA instrument, using platinum crucible. Air atmosphere $\left(50 \mathrm{~mL} / \mathrm{min}\right.$ ) and heating rate of $5^{\circ} \mathrm{C} / \mathrm{min}$. were maintained throughout the experiment.

The XPS surface element analysis of the membranes was performed using a KRATOS AXIS XPS instrument, with $\mathrm{Al} \mathrm{K} \alpha$ excitation radiation (1486.69 eV). The X-ray source was run at $80 \mathrm{~W}$ power. Binding energy was calibrated by using containment carbon (C 1s=286.69 eV). Survey spectrum was run in the binding energy at range of 0-1200 eV and C high-resolution spectrum 1s was collected.

Membrane's mechanical characteristic was determined by tensile strength instrument (model). Membrane was cut as standard (25 x 1.5 $\mathrm{cm}$ ) of tensile strength test specimen form. Both ends of this specimen were clamped on a mechanical machine test then they were pulled and observed on their tensile strength and the elongation percentage.

In determining membrane hydrophilicty, apparent contact angle measurement was conducted with static water sessile drops using the Axisymmetric Drop Shape Analysis Profile (ADSA-P) approach. Swelling parameter of the membranes was calculated based on water uptake content (WCU) using this formula:

$\%$ water uptake $=($ final weight - initial weight)/initial weight $\times 100$

Final weight was the swollen film (soaked for $6 \mathrm{~h}$ in phosphate buffer, $\mathrm{pH}$ 7.4) after diluting the surface water with a filter paper and the initial weight was the dried weight's film.

\section{Urea permeation study}

Transport test was conducted using transport equipment which consists of source phase and acceptor phase. There was membrane in the middle of that phase. Source phase was filled with $50 \mathrm{~mL}$ of urea solution and acceptor phase filler with $50 \mathrm{~mL}$ of phosphate buffer without urea. Transport was conducted for $6 \mathrm{~h}$, in every hour the solution from source phase and acceptor phase was taken as much as $2 \mathrm{~mL}$ and got complex with $\mathrm{p}$-dimethylamine benzaldehyde at $\mathrm{pH}$ 3. The urea concentration permeated by acceptor phase was analyzed using UV-Vis spectrometer at $425 \mathrm{~nm}$ wavelength.

Permeability coefficient was measured using the formula, as follows:

$$
\ln \frac{\mathrm{C}_{0}}{\mathrm{C}_{\mathrm{t}}}=\frac{\text { P.S.t }}{\mathrm{V} . \mathrm{l}}
$$

$\mathrm{C}_{0}$ is the initial concentration in the source solution (donor), $\mathrm{C}_{\mathrm{t}}$ is the solution concentration at $t$ time, $P$ is the permeability coefficient which is the area of contacted membranes $\left(19.1 \mathrm{~cm}^{2}\right), \mathrm{V}$ is the volume of source solution $(50 \mathrm{~mL})$ and 1 is membrane's thickness. 


\section{RESULTS AND DISCUSSION}

Chitosan has two reactive groups, $-\mathrm{NH}_{2}$ group and $-\mathrm{OH}$ which are easily protonated and substituted with other compounds through either cross-linking or grafting process. Chitosan was cross-linked with tripolyphospate (Na-TPP) to increase the active sides that could bind urea through forming hydrogen bond. Cross-linking process began with the addition of strong acid as the catalyst therefore chitosan's $-\mathrm{NH}_{2}$ could be protonated into $-\mathrm{NH}_{3}{ }^{+}$, then $-\mathrm{NH}_{3}{ }^{+}$would bind ionotropically with negative ion (-PO$\left.{ }_{4}{ }^{3-}\right)$ from TPP. Bhumkar and Pokharkar study (2006) stated that in acid $\mathrm{pH}$, TPP compound would be in phosphate state, however, in base $\mathrm{pH}$ there would be $\mathrm{OH}^{-}$ion and phosphate ion in the solution system. This would cause a competition between the two ions to interact with chitosan $\mathrm{NH}_{3}{ }^{+}$. To determine the success of the crosslinking reaction, the functional groups were identified using FTIR spectrometry in shown in Figure. 1.

Chitosan specific absorption on 1573$1595 \mathrm{~cm}^{-1}$ area which was twin absorption of primary amine groups did not appear in CS-TPP spectrum, however, single absorption appeared at $1650 \mathrm{~cm}^{-1}$ wavelength, it indicated that chitosan amine groups changed, from primary into secondary as the result of cross-linking process. This is in accordance with Sarkar et al., study ${ }^{14}$ and Gierszewska and Ostrowska-Czubenko ${ }^{15}$ that stated when chitosan amine groups were protonized and ionotropically cross-linked with TPP groups, it would move the absorption from $1590 \mathrm{~cm}^{-1}$ into $1650 \mathrm{~cm}^{-1}$ area. The success of this cross-link reaction was indicated by strong absorption between $1000-1300 \mathrm{~cm}^{1}$ area. There were two peak of $-\mathrm{PO}_{3}$ group absorption, one peak at $1165 \mathrm{~cm}^{-1}$ and the other one at 1095 $\mathrm{cm}^{-1}$. In addition, there was absorption at $902 \mathrm{~cm}^{-1}$ coming from $-\mathrm{P}-\mathrm{O}$ group.

From FTIR spectra result towards the variation of the added tripolyphosphate concentrations, it showed that there was no significant spectra difference, either from the intensity or absorption strength. From the overall membrane spectra, it was observed that crosslinking process was successfully conducted.

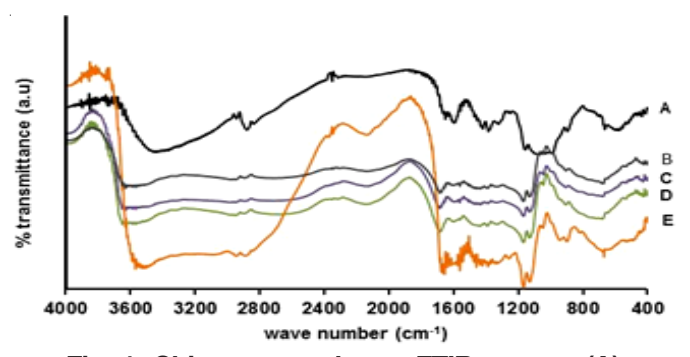

Fig. 1. Chitosan membrane FTIR spectra (A), CS-TPP1(B), CS-TPP2(C), CS-TPP3 (D) and CS-TPP4(E)

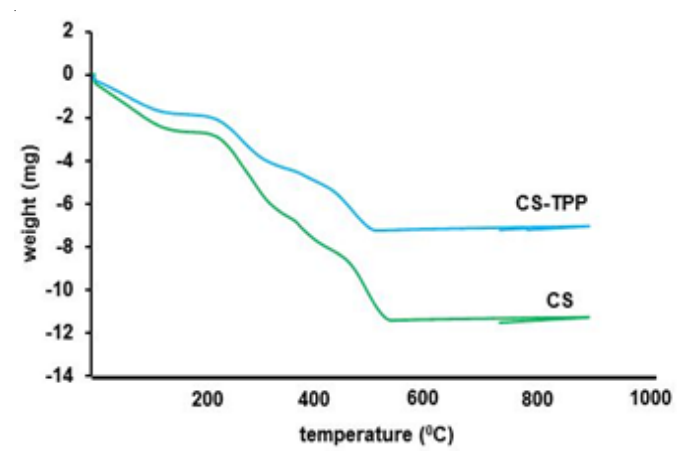

Fig. 2.Thermogram of chitosan membrane and tripolyphosphate crosslinked chitosan membrane

To further reinforce the crosslinking reaction of tripolyphosphate to chitosan, also carried out thermogravimatric analysis as shown in Fig. 2. While comparing their stability, the lower shifts in the decomposition temperature are recorded from 250 to $400^{\circ} \mathrm{C}$ consequently, there is a crosslinking due to the decreasing degree of crystallinity. Thermal decomposition at a sample rate of $-40 \%$ also produce content especially for residual phosphate inorganic material, which indicates the inclusion of TPP significantly into chitosan structure.Thermal decomposition at the sample rate also provided $40 \%$ inorganic content primarly due to phosphate residue in the membrane material, that indicates significant TPP modified into the chitosan structure. This thermogram is in accordance with the thermal analysis results performed by Sarkar et al., 2013.

Further analysis of the success of crosslinking reactions is done by elemental analysis using the XPS method. Elemental composition of the membrane surface based on XPS spectra analysis can be seen in Table 2 . 
Table. 2: Atomic composition of the sample surface which were measured based on XPS spectra data

\begin{tabular}{lcccc}
\hline Surface & $\mathrm{C} \%$ & $\mathrm{O} \%$ & $\mathrm{~N} \%$ & $\mathrm{P} \%$ \\
\hline CS & 78.62 & 16.21 & 3.65 & 0 \\
CS-TPP1 & 72.34 & 21.40 & 2.08 & 1.51 \\
\hline
\end{tabular}

Based on elemental analysis in CS membrane, the composition was decreased both from $\mathrm{C}$ and $\mathrm{N}$ atom. Substitution reaction of polyphosphate group towards chitosan backbone chain caused cross-linked compound molecular weight became bigger. The consequence of that molecular weight addition caused larger divider value, therefore $\mathrm{C}$ percentage decreased by $6.3 \%$ and $\mathrm{N}$ percentage decreased by $1.5 \%$. Phosphate group substitution changed $\mathrm{P}$ and $\mathrm{O}$ atom presence in polymer chain. In chitosane membrane, there was no $\mathrm{P}$ atom, however, in modification membrane $1.51 \%$ of $P$ atom was suspected coming from phosphate group in cross-linking process. The increasing of $\mathrm{O}$ atom composition which showed TPP presence on membrane surface was also the result of cross-linking TPP groups in chitosan backbone chain. Based on that data, it can be concluded that cross-linking reaction occurred between chitosan and tripolyphosphate with amine group substitution, in accordance with $\mathrm{pH}$ condition during the cross-linking reaction process.

\section{Membranes characterization}

The resulting membranes film is thick $\left(20 \pm 10 \times 10^{-2} \mathrm{~mm}\right)$, flexible, and clearly transparent. Based on the water contact angle (Table 3), it showed that the whole CS-TPP modification membrane was more hydrophilic than chitosan membrane. CS-TPP1, CS-TPP2, CS-TPP3 and CSTPP4 monomers had $15^{\circ}, 30^{\circ}, 40^{\circ}$, and $50^{\circ}$ contact angle, respectively. These results indicated that cross-linked triphosphate groups enhanced membrane ability to contact with water. High $-\mathrm{PO}_{3}$ content caused hydrogen bond formation between polymer and water ability became larger ${ }^{15-16}$. As a membrane candidate that will be used in hemodialysis process, water uptake ability is important for the membrane produced in this study. This is mainly associated with membrane removing/ permeation ability towards permeate as the cause of kidney failure, such as urea, creatinine, and glucose that were dissolved in water system. Membrane water uptake percentage was determined by measuring membrane's before and after weigh which was soaked in water for 2,4 , and hours.

Table 3 shows the absorption percentage difference towards water on various membranes. Chitosan membrane had the lowest water uptake which was $100.0 \%$. Cross-linked tripolyphosphate towards chitosan enhanced membrane water uptake ability as $150 \%$. CS-TPP1, CS-TPP2, CSTPP3 , and CS-TPP4 has 251, 245, 247, and 248 $\%$ water uptake percentage, respectivelly.

This result was in accordance with membrane contact angle towards water, CS-TPP1 showed the lowest contact angle. Adding more TPP made the membrane become more hydrophilic. It was possible due to $-\mathrm{PO}_{3}$ groups in modification membrane caused hydrogen binding with the surrounding water increased as well as the increase of free -O group amount which could bond with water molecule. It indicated that a proper balance between hydrophillic-hydrophobic groups in CSTPP membrane was achieved.

Table.3: Contact angle and membrane water uptake percentage

\begin{tabular}{lcc}
\hline Membran type & $\begin{array}{c}\text { Contact } \\
\text { angle }\left(^{\circ}\right)\end{array}$ & $\begin{array}{c}\text { Water Uptake } \\
(\%)\end{array}$ \\
\hline CS & 80 & 100 \\
CS-TPP1 & 15 & 251 \\
CS-TPP2 & 30 & 245 \\
CS-TPP3 & 40 & 247 \\
CS-TPP4 & 50 & 248 \\
\hline
\end{tabular}

Tensile strength test is aimed to determine membrane mechanical strength towards the environment force. The test describes membrane stretch/elasticity strength. A membrane is grouped as elastic if it has high stretching ability when given some force on itself. Elastic membrane is more advantageous than fragile membrane. In the testing process, the test material was pulled with a number of forces until it broke and we obtained the modulus young value. 
From Table 4, membrane strength increased as the cross-linked TPP towards chitosan happened. It can be seen by the increase of membrane modulus young value. This proved that tripolyphosphate groups cross-linking process towards chitosan enhanced membrane tensile and strain strength significantly. The largest membrane strength was in CS-TPP 4 which had higher tensile and strain strength than the other membranes (Fig.3). This value showed that modified membrane had higher elasticity compared with chitosan. CS-TPP membrane tensile strength decreased as of the increase of NaTPP used in crosslinking process.

Table.4: Some modulus young of CS-TPP membranes

\begin{tabular}{lccc}
\hline $\begin{array}{l}\text { Membrane } \\
\text { type }\end{array}$ & $\begin{array}{c}\text { Tensile } \\
\text { strength } \\
\text { (MPa) }\end{array}$ & $\begin{array}{c}\text { Strain } \\
(\%)\end{array}$ & $\begin{array}{c}\text { Modulus } \\
\text { young }\end{array}$ \\
\hline CS & 1.3132 & 18.2822 & 0.0718 \\
A (CS-TPP1) & 0.1225 & 8.6734 & 1.4129 \\
B (CS-TPP2) & 2.3122 & 50.7244 & 4.5583 \\
C (CS-TPP3) & 2.4878 & 53.0999 & 4.6851 \\
D(CS-TPP4) & 5.7273 & 112.9972 & 5.0685 \\
\hline
\end{tabular}

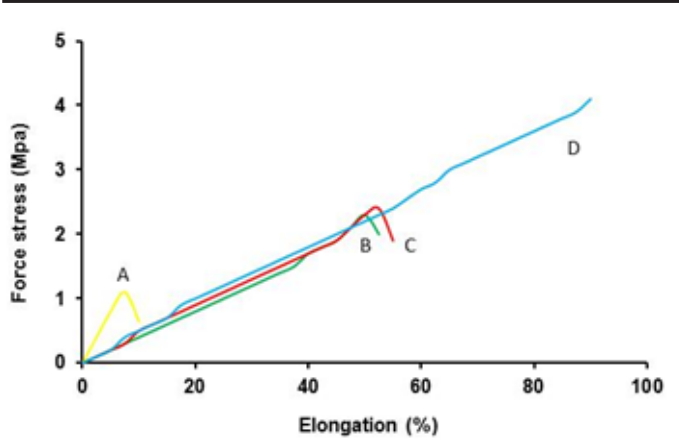

Fig.3. Tensile strength and elongation at broken membrane

\section{Membrane transport ability}

In urea transport process, membrane was placed between two chambers. 500 ppm urea solution in phosphate buffer was placed in the first chamber as source phase. The second chamber was the acceptor phase filled with phosphate buffer. Then, urea in source phase was captured by active phosphate group along in the membrane surface through strong hydrogen bond. Together with the solvent, it diffused into membrane interphase through the pore lanes with relay system by the active sides in the membrane until the end border of membrane interphase which faced acceptor phase and would be released in the acceptor phase. This process occurred continuously until transport process balance reached, wherein membrane could not pass through creatinine.

The data transport showed that CS-TPP membrane with the highest TPP concentration had the highest transport percentage compared with the other membranes. It was possible because of the more TPP added the more active sides that captured permeate, and this was the right distance in capturing and holding target compound (urea) process. The right distance resulted to balanced distance and it implied the percentage of urea transport. In CS-TPP1membrane, transport percentage was $44 \%$ and water uptake was $251 \%$. Wang ${ }^{21}$ stated that right balance between hydrophilic groups and hydrophobic groups resulted into membrane with excellent characteristics, and had high transport ability.

In general, the transport percentage tended to increase in CS-TPP membrane rather than in chitosan membrane. Cross-linking reaction towards chitosan made the produced compound had more regular distance. Phosphate groups that entered the system would attract chitosan molecule to move away from each other, and formed cavity in the polymer which resulted to an ordered distance ${ }^{18,19}$. It was suspected that the distance between monomers in a polymer hold a role in transport process ${ }^{20}$, with a certain and ordered distance, solvent as transport media could optimally diffuse in the membrane and formed right sized cavity as space for target compound. Cross-linked active groups in the surface and membrane pore helped membrane to capture and hold target

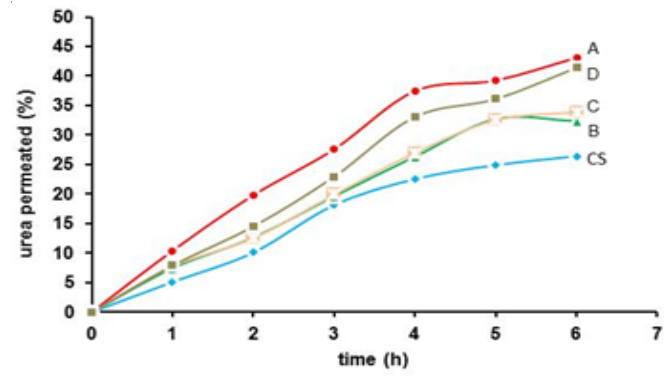

Fig.4. Urea permeation percentage in some CS membranes and CS-TPP 1 (A), CS-TPP2 (B), CS-TPP3 (C), and CS-TPP4 (D) 
compound during transport process to achieve high percentage of transport final result.

\section{CONCLUSION}

Modification of tripolyphosphate groups on chitosan by crosslinking process increases water uptake by $150 \%$, mechanical strength, enhance of percentage urea permeated equal to $10-15 \%$.This indicates that entry of the tripolyphosphate group increases the number of active sites in the chitosan membrane.

\section{ACKNOWLEDGEMENT}

This work was supported by General Directorate of Research, Technology and Higher Education, Indonesia foundation through postdoctoral grant.We wish to express our thanks to the editor and reviewers for their valuable comments.

\section{REFERENCE}

1. Lusiana, R.A., Siswanta, D., and Mudasir, Indones. J. Chem., 2016, 16, 144-150.

2. Wijaya, A. R., Ohde, S., Shinjo, R., Ganmanee, M., and Cohen, M, Arabian J. Chem., 2017.

3. Shaari, N., and S. K. Kamarudin., J. Power Sources, 2015, 289, 71-90.

4. Vanholder, R., Clin. Mater.,1992, 10, 87-133.

5. Yu, X., Shen, L., Zhu, Y., Li, X., Yang, Y., Wang, X., Zhu, M., and Hsiao, B.S., J. Membr. Sci., 2017, 523, 173-184.

6. Huangfu, P., Gong, M., Zhang, C., Yang, C., Zhao, J., and Gong, Y, Colloids and Surf. B, 2009, 71, 268-274.

7. Vimal, S., Abdul Majeed, S., Taju, G., Nambi, K.S.N., Sundar Raj, N., Madan, N, Farook, M.A., Rajkumar, T., Gopinath, D., and Sahul Hameed, A.S., Acta Trop., 2013, 128, 486-493.

8. Sagnella S. and Mai-Ngam, K., Colloids Surf., 2005, 42, 147-155.

9. Li, L., Cheng C., Xiang, T., Tang, M., Zhao, W., Sun, S., and Zhao, C., J.Membr.Sci., 2012, 405-406, 261-274.

10. Lusiana, R.A., Siswanta D., Mudasir dan Hayashita T.,Indo J.Chem, 2013, 13, 262-170.

11. Prashanth, K.V.H and Tharanathan, R.N.,
Carbohydr. Polym., 2005, 341, 169-173.

12. Sarkar, S.D., Farragia, B.L., Dargaville, T.R. and Dhara, S., Mater. Sci. Eng. C, 2013, 33, 1446-1454.

13. Xue, C., and Wilson, I.D., Carbohydr. Polym., 2016, 135, 180-186.

14. Bhumkar and Pokharkar, Pharm. Sci. Technol, 2006, 7 (2), E1-E6.

15. Gierszewska, M., and Ostrowska-Czubenko, J., Carbohydr. Polym., 2016, 153, 501-511

16. Rios, F., Dissertation, New Mexico State University, 2011.

17. Amri, C., , Mudasir, M., Siswanta, D., and Roto, R., Int. J. Biol. Macromol., 2015, 191-196

18. Anitha A., Rejinold, S., Bumgardner, J.D., Nair, S.,V., and Jayakumar R., Chitosan Based Systems for Biopharmaceuticals, John Wiley and Sons, 2012.

19. Buranachai, T., Praphairaksit, N., and Muangsin, N., Pharm. Sci. Technol. Today, 2010, 11, 1128-1137.

20. Balan, V, and Verestiuc, L., Euro.Polym.J., 2014, 53, 171-188.

21. Wang, X.H., Tian, Q., Wang, W., Zhang, C.N., Wang, P., dan Yuan, Z., J. Mater. Sci., 2012, 23, 1663-1674. 